\title{
Left atrial appendage closure using the Watchman device in patients with off-label anatomy: "No man left behind"
}

\author{
Linying Xia \\ Department of Cardiology, The Second Affiliated Hospital of Xi'an Jiaotong University, Xi'an 710004, \\ Shaanxi, China
}

The Watchman (Boston Scientific, Marlborough, MA, USA) occluder is specifically designed to be permanently implanted at or slightly distal to the left atrial appendage (LAA) ostia trapping thrombus before they exit the LAA. With accumulating proof that LAA closure using the Watchman device is effective in reducing the risk of thromboembolism in patients with atrial fibrillation, it is becoming a worldwide predominant occluder. There are five different sizes: 21, 24, 27, 30 and $33 \mathrm{~mm}$, and are labeled for patients with an LAA ostial diameter between 17 and $31 \mathrm{~mm}$ [1]. However, LAA anatomy is highly heterogeneous, with a range of ostia diameters from 5 to $40 \mathrm{~mm}$ [2]. The currently available devices are far from ideal, and in some patients, the conventional practice of LAA closure with a Watchman device could not obtain optimal anatomic results, which requires no peri-device leakage of clinical significance $(>5 \mathrm{~mm}$ ) due to a suboptimal match between the device and a complex LAA morphology [3]. Therefore, the technical challenges of percutaneous LAA occlusion relate to the morphology of LAA, including the number of lobes, and the maximum and minimum of ostia suitable for intervention. An off-label attempt is necessary to widen the indication of Watchman in a complex LAA anatomy in order to leave no man behind. Giant single-lobulated LAA, multilobed LAA and small LAA present a real challenge for conventional Watchman-based LAA closure. Herein, is summarized the latest reports regarding Watchman-based technique innovations, focusing on patients with off-label LAA anatomy.
A single-device LAA occlusion technique concerns the maximum LAA body size suitable for intervention. Therefore, in patients with the single-lobulated LAA and giant ostia $>31 \mathrm{~mm}$, complete closure of the LAA could not be achieved with a single Watchman implantation. Clinically obvious residual flow $>5 \mathrm{~mm}$ in the LAA is associated with an increasing risk of thromboembolism which requires prolonged antithrombotic therapy thereby defeating the purpose of an LAA closure [4]. Accordingly, adequate closure of this giant LAA is essential. In the current issue of the 'Cardiology Journal' [5], a technology note was published reporting three successful attempts using a kissing-Watchman strategy by deploying two Watchman devices adjacently in a kissing-fashion to achieve complete LAA occlusion in patients with singlelobulated LAA and giant ostia ( $>31 \mathrm{~mm})$. Despite a more liberal oversizing technique, favorable anatomic results were achieved in all 3 patients. Image follow-up showed adequate LAA sealing (peri-device leakage $<5 \mathrm{~mm}$ ), without devicerelated thrombosis or obvious device dislocation. There were no major adverse events including device embolism, pericardial tamponade, stroke/ /transient ischemic attack/systemic embolism, death or major bleeding during a 1-year clinical follow-up. This technical innovation shows that a more liberal oversizing technique contributes to greater stability rather than a hazard for device dislocation and provides a reasonable LAA closure strategy in this single-lobulated LAA with giant ostia anatomy.

Address for correspondence: Linying Xia, MD, PhD, Department of Cardiology, The Second Affiliated Hospital of Xi'an Jiaotong University, 157 Xi Wu Road, Xi’an 710004 China, tel: 86-18729908799, e-mail: 467226125@qq.com

Received: 3.09.2019

Accepted: 19.01.2020 
Owing to the variations of LAA anatomy, in terms of number of lobes, it is impossible to completely close LAA with a single Watchman device. The LAA uncovered lobe was deemed to be a nidus for thrombus formation, because of slow blood flow caused by a ridge-like pectinate muscle which separates the LAA into two lobes. Accordingly, adequate closure may require more than a single device in LAA morphology with two outgoing lobes. Liu et al. [6] and Jiang et al. [7], respectively, reported their initial experience on either one-stop or staged double-Watchman implantation. This anatomic structure warranted that the two devices were implanted separately rather than adjacently, reducing the possibility of direct mechanical interaction between the two devices. Sealing both the lobes was essential to effectively reducing the risk of thrombus formation. According to clinical observations and image follow-up, none of the patients had severe complications or major adverse events. Their reports provide a feasible and safe strategy avoiding incomplete LAA closure due to a single Watchman implantation in this bilobulated-LAA anatomy.

According to Food and Drug Administration labelling, it is recommended in patients with a maximal LAA ostial diameter of $17 \mathrm{~mm}$ in order to accommodate the smallest Watchman device (21 mm) currently available for LAA occlusion. Deploying a round occluder into a relatively small ostium may lead to excessive radial force, which could theoretically result in endocardium perforation or device embolization. Additionally, shoulder protrusion of Watchman device out of the LAA may interfere with the surrounding structure (circumflex coronary artery, mitral valve, pulmonary vein). In the issue of 'Journal of Cardiovascular Electrophysiology', Venkataraman et al. [8] reported their experience in 31 out of 32 patients with the mean maximal LAA ostial width range from $14 \mathrm{~mm}$ to $16 \mathrm{~mm}$ who successfully underwent Watchman implantation. Although the maximum compression ratio was numerically greater than manufacturer recommendations (20\%) [9], no adverse events of clinical significance occurred during a 45 day follow-up. Their experience again substantiated the fact that over-compression to some extent is not a hazard for device dislocation. Additionally, moderate shoulder protrusion of the Watchman device would not interfere with the surrounding structure of LAA. This report provides first-hand evidence that Watchman implantation is safe in occluding smaller LAA with a maximal LAA ostial width $<17 \mathrm{~mm}$.
There is a kaleidoscope of variations concerning LAA anatomy in terms of the diameter of ostia and plane of lobes, and no occluder fits them all. While those technical innovations can be useful, a word of caution should be issued since late mechanical interaction is unknown and the number of patients and follow-up duration are limited in all these reports. More intense imaging follow-up may be considered, when dealing with LAAs with these complex anatomic features. The authors mentioned above should be congratulated for their effort in these technical innovations, however, the safety and efficacy of these strategies remain to be verified by larger cohorts. Nevertheless, these technique innovations of LAA closure may overcome the anatomic limitation and potentially widen Watchman-based LAA occlusion indications in all candidates for percutaneous LAA closure.

\section{Conflict of interest: None declared}

\section{References}

1. Sick PB, Schuler G, Hauptmann KE, et al. Initial worldwide experience with the WATCHMAN left atrial appendage system for stroke prevention in atrial fibrillation. J Am Coll Cardiol. 2007; 49(13): 1490-1495, doi: 10.1016/j.jacc.2007.02.035, indexed in Pubmed: 17397680.

2. Ernst G, Stöllberger C, Abzieher F, et al. Morphology of the left atrial appendage. Anat Rec. 1995; 242(4): 553-561, doi: 10.1002/ ar.1092420411, indexed in Pubmed: 7486025.

3. Tzikas A, Holmes DR, Gafoor S, et al. Percutaneous left atrial appendage occlusion: the Munich consensus document on definitions, endpoints and data collection requirements for clinical studies. Europace. 2017; 19(1): 4-15.

4. Lam SC, Bertog S, Sievert H. Incomplete left atrial appendage occlusion and thrombus formation after Watchman implantation treated with anticoagulation followed by further transcatheter closure with a second-generation Amplatzer Cardiac Plug (Amulet device). Catheter Cardiovasc Interv. 2015; 85(2): 321-327, doi: 10.1002/ccd.25456, indexed in Pubmed: 24550125.

5. Xia L, Liu Yi, Tao L. Kissing-Watchman technique applied in single-lobulated left atrial appendage anatomy with giant ostia. Cardiol J. 2020; 27(1): 78-80, doi: 10.5603/CJ.a2019.0070, indexed in Pubmed: 31313275.

6. Liu Z, Yu J, Fang PH, et al. Double device left atrial appendage closure with the WATCHMAN ${ }^{\mathrm{TM}}$. Int J Cardiol. 2015; 187: 281-282, doi: 10.1016/j.ijcard.2015.03.289, indexed in Pubmed: 25838231.

7. Jiang L, Duenninger E, Muenzel M, et al. Percutaneous left atrial appendage closure with complex anatomy by using the staged ,kissing-Watchman' technology with double devices. Int J Cardiol. 2018; 265: 58-61, doi: 10.1016/j.ijcard.2018.05.007, indexed in Pubmed: 29754931.

8. Venkataraman G, Strickberger SA, Doshi S, et al. Short-term safety and efficacy of left atrial appendage closure with the WATCHMAN device in patients with small left atrial appendage ostia. J Cardiovasc Electrophysiol. 2018; 29(1): 17-21, doi: 10.1111/jce.13333, indexed in Pubmed: 28877379.

9. Möbius-Winkler S, Sandri M, Mangner N, et al. The WATCHMAN left atrial appendage closure device for atrial fibrillation. J Vis Exp. 2012(60), doi: 10.3791/3671, indexed in Pubmed: 22395336. 